\title{
INFECCIÓN POR EL COMPLEJO Mycobacterium avium-intracellulare EN PACIENTES CON VIH/SIDA EN UN HOSPITAL PERUANO: UNA SERIE DE CASOS
}

\author{
César Ticona-Huaroto ${ }^{1, a}$, Lilian Astocondor-Salazar ${ }^{1, a}$, Juan Montenegro-Idrogo ${ }^{1,2, b}$, \\ Gustavo Valencia-Mesias ${ }^{1,2, b}$, Jaime Soria ${ }^{1, a}$
}

\begin{abstract}
RESUMEN
Existe poca información sobre la presencia del complejo Mycobacterium avium-intracellulare (MAC) en el Perú. Se describen cinco casos de infección por MAC en pacientes con VIH/SIDA del Hospital Nacional Dos de Mayo, Lima-Perú. Los pacientes presentaron, principalmente, fiebre persistente, diarrea crónica, síndrome consuntivo, pancitopenia y citofagocitosis. En todos ellos se identificó bacilos acido-alcohol resistentes en heces, por lo que recibieron tratamiento antituberculoso. El cultivo de heces fue negativo para Mycobacterium tuberculosis y, posteriormente, en todos se identificó a MAC mediante una prueba molecular (Genotype) en el cultivo de heces. Tres pacientes recibieron tratamiento para MAC luego de la identificación; sin embargo, todos fallecieron. Ante presentaciones similares a lo reportado, se sugiere el uso de métodos de mayor rendimiento (hemocultivo, mielocultivo, pruebas moleculares), así como asociar tempranamente drogas con actividad para MAC al esquema antituberculoso con la intención de mejorar el pronóstico de este grupo de pacientes.
\end{abstract}

Palabras clave: Complejo Mycobacterium avium; VIH-1; Síndrome de inmunodeficiencia adquirida (fuente: DeCS BIREME).

\section{INFECTION BY THE Mycobacterium avium-intracellulare COMPLEX IN PATIENTS WITH HIV/AIDS IN A PERUVIAN HOSPITAL: A SERIES OF CASES}

\begin{abstract}
There is little information on the presence of the Mycobacterium avium-Intracellulare (MAC) complex in Peru. Five cases of MAC infection are described in patients with HIVIAIDS at the National Hospital Dos de Mayo, Lima - Peru. The patients presented, mainly, persistent fever, chronic diarrhea, consumptive syndrome, pancytopenia and citofagocitosis. In all of them, resistant acid-alcohol bacilli were identified in feces, so they received antituberculous treatment. The culture of feces was negative for Mycobacterium tuberculosis and, later, in all cases MAC was identified using a molecular test (genotype) in the culture of feces. Three patients received treatment for MAC right after identification; however, they all died. Before presentations similar to the reported, we suggest the use of higher performance methods (blood culture, myeloculture, molecular tests), as well as early associating drugs with activity for MAC to antitubercular scheme with the intention of improving the prognosis of this group of patients.
\end{abstract}

Key words: Mycobacterium avium complex; HIV-1; Acquired immunodeficiency syndrome (source: MeSH NLM).

\section{INTRODUCCIÓN}

\section{El complejo Mycobacterium avium-intracellulare} (MAC) está comprendido por las especies M. avium у $M$. intracellulare. Dichas especies están distribuidas ampliamente en el medioambiente -principalmente en el agua y el suelo-, siendo la inhalación y/o ingesta sus probables mecanismos de transmisión (1). En el paciente $\mathrm{VIH}$ coinfectado por MAC se observa una alta frecuencia de colonización y compromiso gastrointestinal, por lo que se postula que sería la mucosa intestinal la vía principal de ingreso (2). El MAC es la principal causa de infección por micobacterias no tuberculosas (MNT) en el paciente con $\mathrm{VIH} / \mathrm{SIDA}{ }^{(2)}$.

En la época previa al tratamiento antirretroviral (TAR), la incidencia de MAC en personas con VIH/SIDA era hasta 25 veces mayor que en la actualidad, principalmente en países de altos ingresos económicos ${ }^{(3)}$. En países de mediano y bajo ingreso la prevalencia ha sido poco estudiada ${ }^{(3)}$.

\footnotetext{
Servicio de Medicina en Enfermedades Infecciosas y Tropicales, Hospital Nacional Dos de Mayo. Lima, Perú.

Facultad de Medicina, Universidad Nacional Mayor de San Marcos. Lima, Perú.

Médico especialista en Medicina de Enfermedades Infecciosas y Tropicales; ${ }^{\mathrm{b}}$ médico cirujano.

Recibido: 04/11/2016 Aprobado: 22/03/2017 En línea: 28/06/2017
}

Citar como: Ticona-Huaroto C, Astocondor-Salazar L, Montenegro-Idrogo J, Valencia-Mesias G, Soria J. Infección por el complejo Mycobacterium avium-intracellulare en pacientes con VIH/SIDA en un hospital peruano: una serie de casos. Rev Peru Med Exp Salud Publica. 2017;34(2):323-7. doi: 10.17843/rpmesp.2017.342.2476 
La infección por MAC en pacientes con inmunosupresión severa por VIH/SIDA se manifiesta, principalmente, de forma diseminada; en otras enfermedades que también comprometen la inmunidad celular, su presentación es más frecuente en el pulmón, piel y tejido blando ${ }^{(4)}$.

La presentación clínica en el paciente con VIH/SIDA se caracteriza por compromiso sistémico, fiebre, diarrea, dolor abdominal, anemia severa, leucopenia y elevación de fosfatasa alcalina ${ }^{(2)}$.

A nivel nacional solo se han reportado dos casos confirmados de infección por MAC en pacientes con $\mathrm{VIH} /$ SIDA ${ }^{(5,6)}$. Este es un reporte de cinco casos de infección por MAC en pacientes con VIH/SIDA hospitalizados entre los años 2015 y 2016 en el Hospital Nacional Dos de Mayo (HNDM), Lima - Perú. El propósito de este reporte es contribuir con información para un reconocimiento temprano de esta patología que podría no ser tan infrecuente en el país.

\section{SERIE DE CASOS}

Entre los años 2015 y 2016, tres pacientes varones y dos mujeres, de edades comprendidas entre los 20 y 40 años, procedentes de la ciudad de Lima, iniciaron su atención en el Servicio de Infectología del HNDM, en estado avanzado de infección por VIH (CD4 inicial < 50 cel $/ \mu \mathrm{L})$. Todos tuvieron un cuadro clínico caracterizado por diarrea crónica severa como síntoma principal, fiebre persistente y síndrome consuntivo severo. Así, se consideró como agente causal al Mycobacterium tuberculosis (MTB) debido al hallazgo de bacilos ácidoalcohol resistentes (BAAR) en muestras de heces, único resultado microbiológico positivo. Otros resultados de los exámenes auxiliares presentaron hipoalbuminemia severa, elevación marcada de fosfatasa alcalina y vitamina B12 (promedio 690 U/L y 1600 pg/mL, respectivamente), pancitopenia con anemia y trombocitopenia severa, y citofagocitosis en el mielograma de manera similar en todos los casos (Tabla 1).

Por las características basales, el cuadro clínico y los hallazgos de laboratorio descritos, todos los pacientes fueron tratados para coinfección VIH/TB durante su hospitalización inicial, e iniciaron el tratamiento antituberculoso con drogas de primera línea. Pese a la terapia instalada, dos pacientes fallecieron durante las primeras semanas del tratamiento. Los otros tres pacientes presentaron una evolución inicialmente favorable con el tratamiento antituberculoso, por lo que continuaron con evaluación ambulatoria e iniciaron el TAR. Aproximadamente, al cuarto mes de tratamiento antituberculoso, los pacientes reingresaron por reaparición de los síntomas iniciales con deterioro clínico progresivo, por lo que se consideró dentro de los posibles diagnósticos a una infección por tuberculosis drogoresistente o la manifestación del síndrome de reconstitución inmune, entre otras etiologías posibles.

Finalmente, se identificó, mediante la prueba molecular Genotype®MTBDRplus (Hain LifeScience, Nehren, Alemania), al MAC en los cultivos de heces de los cinco pacientes. Los tres pacientes sobrevivientes obtuvieron la identificación durante su última hospitalización, por lo que recibieron tratamiento para MAC con claritromicina, etambutol, rifampicina y amikacina; sin embargo, la evolución fue tórpida y todos fallecieron. Uno de los pacientes de evolución y fallecimiento rápido, recibió tratamiento para MAC de manera empírica por la alta sospecha debido a la similitud con los casos anteriores. Solo se realizó necropsia a un paciente, en el que se demostró la infección diseminada mediante el aislamiento microbiológico y molecular de MAC en tejido pulmonar, hepático y de bazo. La identificación mediante Genotype fue realizada en el laboratorio de biología molecular del Instituto Nacional de Salud.

\section{DISCUSIÓN}

La prevalencia de infección por MAC se desconoce en Latinoamérica y el Perú ${ }^{(3)}$. La enfermedad diseminada como infección oportunista en pacientes $\mathrm{VIH}$ se reporta escasamente en nuestra región, concentrándose en Brasil con una prevalencia aproximada del $8 \%{ }^{(7)}$, en contraste con países de alto ingreso en la era pre TAR ${ }^{(3)}$. Esta diferencia se explicaría por la posible protección que brindaría una infección previa por MTB y la vacuna BCG ${ }^{(8)}$, o por un subdiagnóstico de esta condición.

A nivel nacional, hasta el 2002, no se había descrito al MAC dentro de las MNT aisladas en pacientes con $\mathrm{VIH}^{(9)}$; con esta serie, sumarian siete los casos reportados hasta la fecha. Esto, probablemente, responda a las mejores técnicas de identificación del laboratorio de referencia nacional.

Los factores de riesgo presentes en todos los pacientes fueron similares a los reportados en la literatura: adultos jóvenes, conteo de CD4 $<50 \mathrm{cel} / \mu \mathrm{L}$, y ausencia de TAR ${ }^{(2,4)}$. El cuadro clínico de infección por MAC se presentó antes del uso de TAR en todos los casos. No obstante, un cuadro semejante se produce también por MTB ${ }^{(7)}$, siendo la única micobacteria aislada en el mismo contexto clínico en algunos países en desarrollo ${ }^{(10)}$.

Tres de los pacientes tuvieron una respuesta inicial favorable al esquema antituberculoso, suceso que se observa en el $50 \%$ de pacientes con MAC pulmonar (11). En este contexto, el inicio del TAR en presencia de una infección no controlada podría explicar el compromiso sistémico en lugar de uno localizado que se esperaría ver en casos de 
Tabla 1. Características de pacientes con infección VIH y MAC en el Hospital Nacional Dos de Mayo

\begin{tabular}{|c|c|c|c|c|c|}
\hline Características & Caso 1 & Caso 2 & Caso 3 & Caso 4 & Caso 5 \\
\hline Edad (años) & 26 & 23 & 20 & 33 & 39 \\
\hline CD4 inicial (cel/uL) & 9 & 11 & 17 & 23 & 4 \\
\hline CD8 inicial (cel/uL) & 2148 & 163 & 839 & 232 & 122 \\
\hline CV inicial (cop/uL) & 827084 & 554016 & 362305 & 640064 & 313019 \\
\hline \multicolumn{6}{|l|}{ Terapia antirretroviral } \\
\hline Duración (meses)* & 6 & 4 & 4 & 6 & No \\
\hline Esquema & $\mathrm{TDF}+3 \mathrm{TC}+\mathrm{EFV}$ & TDF+3TC+EFV & $\mathrm{TDF}+3 \mathrm{TC}+\mathrm{EFV}$ & $\mathrm{TDF}+3 \mathrm{TC}+\mathrm{EFV}$ & No \\
\hline \multicolumn{6}{|l|}{ Hemograma } \\
\hline $\mathrm{Hb}(\mathrm{g} / \mathrm{L})[$ Hto (\%)] & $7,3[21,6]$ & $7,8[23,3]$ & $7,5[22,3]$ & $6,9[22,0]$ & $6,5[19,1]$ \\
\hline Leu $\left(\operatorname{cel} \times \mathrm{mm}^{3}\right)[\operatorname{Linf}(\%)]$ & $2120[8]$ & 2260 [12] & $580[19]$ & $5200[8]$ & $2280[11]$ \\
\hline Plaquetas $\left(x \mathrm{~mm}^{3}\right)$ & 20000 & 87000 & 25000 & 84000 & 123000 \\
\hline \multirow{3}{*}{ Mielograma } & $\begin{array}{c}\text { - Citofagocitosis, } \\
\text { PR }\end{array}$ & $\begin{array}{c}\text { - Citofagocitosis, } \\
\text { PR }\end{array}$ & $\begin{array}{c}\text { - Citofagocitosis, } \\
\text { PR }\end{array}$ & $\begin{array}{c}\text { - Citofagocitosis, } \\
\text { PR }\end{array}$ & $\begin{array}{c}\text { - Citofagocitosis, } \\
\text { PR }\end{array}$ \\
\hline & - Hemosiderina $\uparrow$ & - Hemosiderina N & - Hemosiderina $\uparrow$ & - Megaloblastosis & $\begin{array}{l}\text { - Predominio } \\
\text { mieloide }\end{array}$ \\
\hline & $\begin{array}{l}\text { - Maduración } \\
\text { mieloide detenida }\end{array}$ & - Megaloblastosis & $\begin{array}{c}\text { - Predominio } \\
\text { mieloide } \\
\text { - Presencia BAAR }\end{array}$ & & \\
\hline Ácido fólico (ng/mL) & 0,64 & 2,62 & -- & -- & 1,15 \\
\hline Vitamina B12 (pg/mL) & 1820 & 1497 & -- & 1322 & 1863 \\
\hline Hierro sérico $(\%)$ & 41 & 25 & -- & -- & 78 \\
\hline Transferrina (mg/dL) & 105 & 150 & -- & -- & 116 \\
\hline Fibrinógeno (mg/dL) & 150 & 709 & 187 & 233 & 333 \\
\hline Triglicéridos (mg/dL) & 82,2 & 154 & 273,3 & 73 & 110 \\
\hline $\mathrm{DHL}(\mathrm{UI} / \mathrm{L})$ & 801 & 955 & 576 & 593 & 896 \\
\hline Fosfatasa alcalina (U/L) & 678 & 693 & 821 & 593 & 705 \\
\hline Albúmina sérica (gr/dl) & 1,33 & 1,91 & 2,1 & 1,11 & 2,59 \\
\hline Ferritina $(\mathrm{ng} / \mathrm{mL})$ & -- & 1650 & -- & -- & $>1650$ \\
\hline Baciloscopía positiva† & $\begin{array}{l}\text { Heces/pulmón } \\
\text { Hígado/bazo }\end{array}$ & Heces & Heces/esputo/MO & Heces & Heces \\
\hline $\begin{array}{l}\text { Tiempo de positividad } \\
\text { (meses) } \ddagger\end{array}$ & 2 & 2 & 2 & 3 & 3 \\
\hline Genotype (muestra) & $\begin{array}{c}\text { M. avium } \\
\text { (cultivo heces) }\end{array}$ & $\begin{array}{l}\text { M. intracellulare } \\
\text { (cultivo heces) }\end{array}$ & $\begin{array}{c}\text { M. avium } \\
\text { (cultivo heces) }\end{array}$ & $\begin{array}{c}\text { M. avium } \\
\text { (cultivo heces) }\end{array}$ & $\begin{array}{c}\text { M. avium } \\
\text { (cultivo heces/ } \\
\text { aspirado gástrico) }\end{array}$ \\
\hline \multicolumn{6}{|l|}{ Tratamiento } \\
\hline Inicial (TB) & HRZE & HRZE & HRZE & HRZE & HRZE \\
\hline MAC & $\begin{array}{c}\text { CLR + E + R } \\
+ \text { AMK }\end{array}$ & $\begin{array}{c}\text { CLR + E + R } \\
\quad+\text { AMK }\end{array}$ & $\mathrm{CLR}+\mathrm{E}+\mathrm{R}+\mathrm{AM}$ & -- & $\begin{array}{c}\mathrm{CLR}+\mathrm{E}+\mathrm{R}+ \\
\mathrm{AMK}\end{array}$ \\
\hline Evolución & $\begin{array}{c}\text { Fallecido al } 14 .^{\circ} \\
\text { día de tratamiento } \\
\text { MAC }\end{array}$ & $\begin{array}{c}\text { Recidivas } \\
\text { intermitentes } \\
2^{\circ} \text { reingreso } \\
\text { Fallecido al } \\
2^{\circ} \text { mes de } \\
\text { tratamiento MAC }\end{array}$ & $\begin{array}{l}2^{\circ} \text { reingreso. } \\
\text { Fallecido a los } \\
6 \text { meses de } \\
\text { tratamiento MAC }\end{array}$ & $\begin{array}{c}\text { Fallecido } \\
10 .^{\circ} \text { día de } \\
\text { hospitalización, } \\
\text { sin tratamiento } \\
\text { MAC }\end{array}$ & $\begin{array}{c}\text { Fallecido al } 12 .^{\circ} \\
\text { día de tratamiento } \\
\text { MAC }\end{array}$ \\
\hline
\end{tabular}

* Duración del último esquema de TARV previo a última hospitalización. El caso 5 inició TARV en la última hospitalización.

† Las baciloscopías de heces de los casos 1, 2 y3 fueron de la hospitalización inicial.

$\ddagger$ Tiempo desde la obtención de la muestra hasta el resultado de tipificación. Caso 4 fue post mortem.

TAR: terapia antirretroviral; TDF: tenofovir; 3TC: lamivudina; EFV: efavirenz; Hb: hemoglobina; Hto: hematocrito; Leu: leucocitos; Linf: linfocitos; PR: plasmocitosis reactiva; N: normal; BAAR: bacilo acido-alcohol resistente; DHL: deshidrogenasa láctica; MO: médula ósea; HRZE: esquema de primera línea antituberculosis; CLR: claritromicina; E: etambutol; R: rifampicina; AMK: amikacina; MAC: M. avium complex. 
síndrome inflamatorio de reconstitución inmune asociado a MAC ${ }^{(2)}$. Esto habría favorecido la presentación severa y el desenlace fatal posterior al inicio del TAR, similar al reportado por Cordero et al. ${ }^{(5)}$. Asimismo, el valor elevado de vitamina B12 se correlacionaría con severidad y mortalidad ${ }^{(12)}$, y el de ferritina con una respuesta inflamatoria intensa incluyendo síndrome hemofagocítico ${ }^{(13)}$.

En el Perú, la estrategia de detección de micobacterias comprende la baciloscopía, los cultivos en medios sólidos (Lowenstein-Jensenm, Ogawa y agar 7H10) y líquidos (MGIT y MODS) ${ }^{(14)}$, razón que explicaría el bajo aislamiento de MAC, pues, incluso en enfermedad diseminada, la probabilidad de resultados negativos para MAC, tanto en baciloscopia como en cultivos de esputo, es mayor en comparación con el MTB ${ }^{(2)}$. Además, los medios sólidos presentan porcentajes de positividad menores y tiempos de detección más largos que los medios líquidos (15). Por otro lado, el hemocultivo y cultivo de medula ósea tienen mejor rendimiento, siendo en varios casos el hemocultivo la única prueba positiva que determina el diagnóstico ${ }^{(7)}$. Una vez detectado por estos medios de cultivo, las pruebas moleculares pueden identificar definitivamente a MAC en cuestión de horas. Igualmente, mediante PCR se puede detectar directamente en sangre ${ }^{(1)}$.

En los casos de este estudio se logró identificar diversas especies del complejo -entre ellas al Mycobacterium intracellular-; sin embargo, el cuadro clínico fue similar en todos los pacientes. Solo un caso tuvo aislamiento de MAC en varios tejidos (mediante necropsia), resultado que sumado a los hallazgos clínicos confirmaron el diagnóstico de enfermedad diseminada por MAC. En los demás casos, por las características clínicas y evolución compatibles, además del aislamiento de MAC en heces, se consideró el mismo diagnóstico.

La terapia de elección tiene como base un macrólido (claritromicina, azitromicina) en combinación con etambutol, prefiriéndose usar claritromicina por la rápida esterilización de los hemocultivos. Se desconoce la susceptibilidad a claritromicina de las cepas aisladas y si esto influyó en el pronóstico. En determinadas situaciones se puede considerar la adición de rifampicina y un aminoglucósido (amikacina). El beneficio de añadir rifamicinas es controversial, recomendándose administrar por un tiempo no mayor a tres meses, en fase aguda de enfermedad, o en presencia de elevada carga bacilar ${ }^{(1)}$. Esta combinación terapéutica se usó en cuatro de los casos reportados, pero el diagnóstico tardío y la resistencia a etambutol y rifampicina presentada en tres de los aislamientos habrían favorecido el fracaso del tratamiento.

Considerando la mayor prevalencia del M. tuberculosis, es común pensar en tuberculosis drogorresistente frente a una mala evolución con el tratamiento de primera línea. Empero, es cada vez mayor el reporte de MNT en pacientes previamente considerados fracasos al tratamiento antituberculoso (11). En Brasil se ha reportado que el $79 \%$ de MNT recibieron tratamiento antituberculoso antes de su diagnóstico definitivo. Otro estudio realizado en Irán reportó que el 30\% de los casos sospechosos de tuberculosis drogoresistente fueron finalmente MNT ${ }^{(11)}$. Es probable que en nuestro país la infección por MAC esté siendo subdiagnosticada.

La infección por MAC debe ser considerada entre los pacientes con infección por VIH y conteo de células CD4 menor a 50cel/uL que presenten manifestaciones clínicas predominantemente gastrointestinales, asociadas a compromiso hematológico, en presencia de BAAR. Por ello, sugerimos usar métodos diagnósticos de mayor rendimiento (hemocultivos, mielocultivo, pruebas moleculares), así como asociar tempranamente drogas con actividad para MAC al tratamiento antituberculoso con la intención de mejorar el pronóstico de este grupo de pacientes.

Contribuciones de autoría: CTH, LAS y JS contribuyeron en la concepción de artículo; CTH realizó el diseño del estudio; JMI y JVM realizaron la recolección de datos. CTH y JS realizaron la revisión crítica del artículo. CTH, LAS, JMI y JVM realizaron la redacción de la versión final del artículo. Todos los autores aprobaron la versión final del artículo.

Fuentes de financiamiento: autofinanciado.

Conflictos de interés: los autores declaran no tener ningún conflicto de interés.

\section{REFERENCIAS BIBLIOGRÁFICAS}

1. Gordin FM, Horsburgh R. Mycobacterium avium Complex. En: Bennett JE, Dolin R, Blaser MJ, editores. Mandell, Douglas, and Bennett's principles and practice of infectious diseases. 8a ed. Philadelphia: Saunders Elsevier; 2015. p. 2832-2844.

2. CortiM,Palmero D.Mycobacterium avium complex infection in HIV/AIDS patients.
Expert Rev Anti Infect Ther. 2008;6(3)35163. doi: 10.1586/14787210.6.3.351

3. Coelho L, Veloso VG, Grinsztejn B, Luz PM. Trends in overall opportunistic illnesses, Pneumocystis carinii pneumonia, cerebral toxoplasmosis and Mycobacterium avium complex incidence rates over the 30 years of the HIV epidemic: a systematic review.
Braz J Infect Dis. 2014;18(2):196-210. doi: 10.1016/j.bjid.2013.10.003

4. Henkle E, Winthrop KL. Nontuberculous mycobacteria infections in immunosuppressed hosts. Clin Chest Med. 2015;36(1):91-9. doi: 10.1016/j.ccm.2014.11.002

5. Mejía Cordero F, Guevara Miranda J, Huerta Mercado J, Legua Leiva P, Seas 
Ramos C. Compromiso intestinal por Mycobacterium avium en un paciente con VIH/SIDA. Rev Gastroenterol Peru. 2014;34(1):59-61.

6. Carrasco J, Soto L, Samalvides F, Asencios L, Quispe N, Valencia E. Infección pulmonar por Mycobacterium avium en paciente VIH/SIDA: primer reporte en Perú. Rev Peru Med Exp Salud Pública. 2014;31(1):156-9.

7. Nakatani SM, Messias-Reason IJ, Burger M, Cunha CA. Prevalence of Mycobacterium avium and Mycobacterium tuberculosis in blood cultures of Brazilian AIDS patients after introduction of highly active retroviral therapy. Braz J Infect Dis. 2005;9(6):459-63.

8. Trnka L, Danková D, Svandová E. Six years' experience with the discontinuation of BCG vaccination. 4. Protective effect of BCG vaccination against the Mycobacterium avium intracellulare complex. Tuber Lung Dis. 1994;75(5):348-52. doi: 10.1016/0962-8479(94)90080-9
9. Quispe N, Asencios L, Vasquez L, Leo E, Lecca L, Llanos-Zavalaga F. Detección de micobacterias tuberculosas y no tuberculosas en pacientes VIH-TB, 1999-2001. Lima: Rev Peru Med Exp Salud Pública; 2002;19(Suppl1):S6.

10. Rivera-Olivero I, Nohra C, Guevara R, Pestana L, Comegna M, De Waard J. Fiebre de origen desconocido en pacientes con SIDA en el Hospital "Jose Maria Vargas" Caracas-Venezuela: ¿Mycobacterium avium complex (MAC) o tuberculosis diseminada? Bol Venez Infectol. 2009; 20(1):24-7.

11. Raju RM, Raju SM, Zhao Y, Rubin EJ. Leveraging advances in tuberculosis diagnosis and treatment to address nontuberculous Mycobacterial disease. Emerg Infect Dis. 2016;22(3):365-9. doi: $10.3201 /$ eid2203.151643

12. Arendt JF, Nexo E. Unexpected high plasma cobalamin: proposal for a diagnostic strategy. Clin Chem Lab Med. 2013;51(3):489-96. doi: $10.1515 / \mathrm{cclm}-2012-0545$

13. Moore C Jr, Ormseth M, Fuchs H. Causes and significance of markedly elevated serum ferritin levels in an academic medical center. J Clin Rheumatol. 2013;19(6):324-8. doi: 10.1097/RHU.0b013e31829ce01f

14. Estrategia Sanitaria Nacional de Prevención y Control de la Tuberculosis. Norma Técnica de Salud para La Atención Integral de las Personas afectadas por Tuberculosis [Internet]. Lima: MINSA; 2013 [citado 10 ene 2017]. Disponible en: ftp://ftp2. minsa.gob.pe/normaslegales/2013/ RM715_2013_MINSA.pdf

15. Abe C, Hosojima S, Fukasawa Y, Kazumi Y, Takahashi M, Hirano K, et al. Comparison of MB-Check, BACTEC, and egg-based media for recovery of mycobacteria. J Clin Microbiol. 1992;30(4):878-81.

Correspondencia: César Ticona.

Dirección: Rio Huaura 319, Pueblo Libre. Lima, Perú.

Teléfono: (+51) 993560273

Correo electrónico: cticonamd@gmail.com

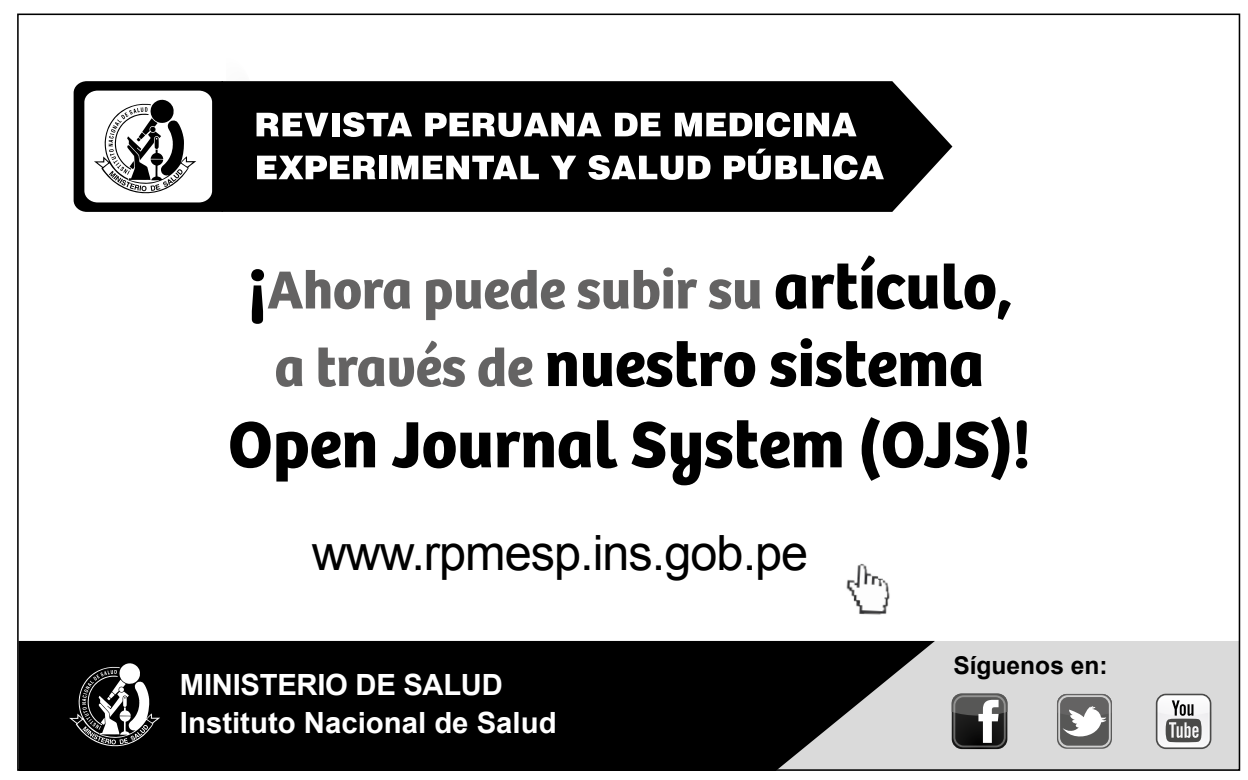

